\title{
$D$-meson observables in heavy-ion collisions at LHC with EPOSHQ model
}

\author{
Vitalii Ozvenchuk ${ }^{1, \star}$, Joerg Aichelin ${ }^{2}$, Pol-Bernard Gossiaux², Benjamin Guiot ${ }^{3}$, Mar- \\ lene Nahrgang ${ }^{2}$, and Klaus Werner ${ }^{2}$ \\ ${ }^{1}$ Institute of Nuclear Physics, Polish Academy of Science, Krakow, Poland \\ ${ }^{2}$ Subatech, UMR 6457, IN2P3/CNRS, Universite de Nantes, Ecole des Mines de Nantes, Nantes, France \\ ${ }^{3}$ Universidad Tecnica Federico Santa Maria, Valparaiso, Chile
}

\begin{abstract}
We study the propagation of charm quarks in the quark-gluon plasma (QGP) created in ultrarelativistic heavy-ion collisions at LHC within EPOSHQ model. The interactions of heavy quarks with the light partons in ultrarelativistic heavy-ion collisions through the collisional and radiative processes lead to a large suppression of final $D$ meson spectra at high transverse momentum and a finite $D$-meson elliptic flow. Our results are in a good agreement with the available experimental data.
\end{abstract}

\section{Introduction}

At LHC the QGP is produced in ultrarelativistic heavy-ion collisions and the nuclear modification factor, $R_{A A}$, and the elliptic flow, $v 2$, of $D$ mesons are measured [1,2]. The $R_{A A}$ of intermediate- and high- $p_{T} D$ mesons is significantly below unity. It indicates a large energy loss of high- $p_{T}$ heavy quarks in the QGP. Many theoretical models describing the $D$-meson observables in heavy-ion collisions [36]. It remains, however, a challenge to describe $R_{A A}$ and $v_{2}$ within the same model input.

In this contribution we present the nuclear modification factor and the elliptic flow of $D$ mesons in heavy-ion collisions at LHC calculated using the different iterations of the EPOSHQ model, in particular, MC@sHQ+EPOS2, MC@sHQ+EPOS3 and EPOSHQ models.

\section{The model}

MC@sHQ+EPOS2 model couples a Monte Carlo propagation of heavy quarks [7], MC@sHQ, to the 3+1 dimensional ideal fluid dynamical evolution of the QGP from EPOS2 initial conditions [8], which combine perturbative QCD (pQCD) calculations of the hard scattering with the Gribov-Regge theory. In MC@sHQ+EPOS3 model we couple MC@sHQ with the recent upgrade EPOS3 that includes a viscous fluid dynamical evolution, based on [9].

We initialize the heavy quarks at the original nucleon-nucleon scattering points according to the $p_{T}$-distribution from pQCD results in fixed order plus next-to-leading logarithm (FONLL) [10]. In EPOSHQ model we implement the heavy quarks directly from EPOS3 initial conditions with initial

^e-mail: Vitalii.Ozvenchuk@ifj.edu.pl 
state shadowing included. The heavy quarks in EPOS3 can be produced during the spacelike cascade, the born process and the partonic shower. Heavy quarks interact with plasma partons by either elastic or radiative collisions. The evolution of heavy quarks is described by the Boltzmann equation. The heavy quarks form hadrons via coalescence or fragmentation on the hypersurface of constant temperature $T=155 \mathrm{MeV}$.

\section{The nuclear modification factor and elliptic flow}

We perform 10000 heavy-quark events per EPOS2 ideal or EPOS3 viscous fluid dynamical event. In Figs. 1 and 2 we show our results for $D$-meson nuclear modification factor and elliptic flow in heavyion collisions at LHC energies within MC@sHQ+EPOS3 model (red lines) as well as the comparison to the MC@sHQ+EPOS2 results (blue lines) taken from [11]. In each model we perform calculations

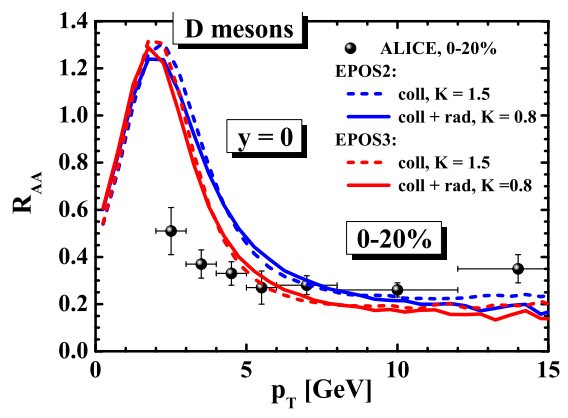

Figure 1. $D$-meson nuclear modification factor calculated in MC@sHQ+EPOS2 model (blue lines) in comparison to the MC@sHQ+EPOS3 results (red lines). Experimental data are taken from [1].

for two scenarios, with purely collisional energy loss (dashed lines) or with collisional+radiative energy loss (solid lines). Both models show a reasonable agreement with the experimental data for the $R_{A A}$ of $D$ mesons at $p_{T}>5 \mathrm{GeV}$. The larger suppression of $D$-meson yield is seen for the

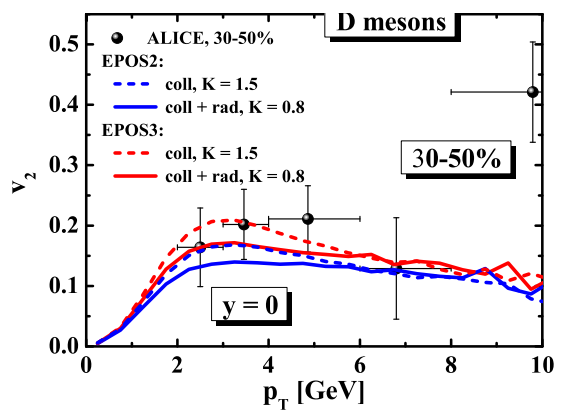

Figure 2. $D$-meson elliptic flow calculated in MC@ $\mathrm{sHQ}+\mathrm{EPOS} 2$ model (blue lines) in comparison to the MC@sHQ+EPOS3 results (red lines). Experimental data are taken from [2].

MC@ $@$ SHQ+EPOS3 results at intermediate $p_{T}$. If the initial state shadowing is not included the results from both models are overestimated at low $p_{T}$. MC@sHQ+EPOS2 and MC@sHQ+EPOS3 results for the elliptic flow of $D$ mesons are in a good agreement with the experimental data for the whole range of $p_{T}$. MC@SHQ+EPOS3 results show the enhancement of $v_{2}$ at intermediate $p_{T}$ in comparison 
to MC@sHQ+EPOS2 results. The final elliptic flow of $D$ mesons can be sensitive to the $D$-meson rescattering in the hadronic phase [12], we need to include the hadronic contribution for further study.

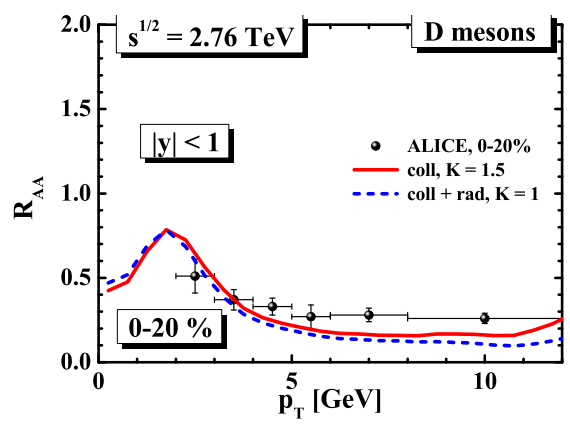

Figure 3. $D$-meson $R_{A A}$ obtained within EPOSHQ model in comparison to the experimental data [1].

In Fig. 3 we present the $D$-meson nuclear modification factor calculated in EPOSHQ model for the central heavy-ion collisions at LHC. The calculations show a good agreement with the experimental data for whole range of $p_{T}$. For two scenarios (pure collisional and collisional+radiative) we obtained almost the same behavior. The presence of the initial state shadowing helped us to describe well the $R_{A A}$ of $D$ mesons at low $p_{T}$.

\section{Conclusions}

In this contribution we have shown the results for $D$-meson observables in ultrarelativistic heavyion collisions at LHC calculated within EPOSHQ model. EPOSHQ model is able to describe the nuclear modification factor and elliptic flow of $D$ mesons in heavy-ion collisions at LHC energies with the same initial setup. For the central lead-lead collisions the importance of including initial state shadowing was presented.

We thank Jan Steinheimer, Iurii Karpenko and Andrzej Rybicki for fruitful discussions. This work was supported by the Project TOGETHER (Pays de la Loire) and by the National Science Center, Poland under Grant No. 2014/14/E/ST2/00018.

\section{References}

[1] B. Abelev et al., JHEP 09, 112 (2012)

[2] B. Abelev et al., Phys. Rev. Lett. 111, 102301 (2013)

[3] M. Nahrgang et al., Nucl. Phys. A 931, 575 (2014)

[4] T. Song et al., Phys. ReV. C 93, 034906 (2016)

[5] S. K. Das et al., Phys. Lett. B 747, 260 (2015)

[6] S. Cao et al., Phys. Rev. C 88, 044907 (2013)

[7] P.-B. Gossiaux et al., Phys. Rev. C 78, 014904 (2008)

[8] K. Werner et al., Phys. Rev. C 82, 044904 (2010)

[9] I. Karpenko et al., Comput. Phys. Commun. 185, 3016 (2014)

[10] M. Cacciari et al., JHEP 10, 137 (2012) 
[11] M. Nahrgang M et al., Phys. Rev. C 89, 014905 (2014)

[12] V. Ozvenchuk et al., Phys. Rev. C 90, 054909 (2014) 